FORMATION Formation emploi

Revue française de sciences sociales

135 | Juillet-Septembre 2016

L'économie verte : rupture ou adaptation de la

formation et de l'emploi ?

\title{
Ruptures ou ajustements provoqués entre pratiques agricoles et enseignement de ces pratiques
}

Implantation et gouvernance de la réforme " Produire autrement » Ruptures or adjustments between agricultural practices and teaching these practices - Implementation and governance of the political reform "Alternatives agricultural Productions"

Völlige Umstellung oder erzwungene Anpassungen der. Durchsetzung und Governance der Reform « Anders produzieren »

Rupturas o ajustes provocados entre prácticas agrícolas y enseñanza de esas prácticas. Implantación y gobernancia de la reforma. "Producir de otra manera"

Jean-François Métral, Paul Olry, Marie David, Fanny Chrétien, Philippe Prévost, Nadia Cancian, Nathalie Frère et Laurence Simonneaux

\section{(2) OpenEdition} Journals

\section{Édition électronique}

URL : http://journals.openedition.org/formationemploi/4856

DOI : $10.4000 /$ formationemploi.4856

ISSN : 2107-0946

Éditeur

La Documentation française

Édition imprimée

Date de publication : 12 octobre 2016

Pagination : 53-74

ISSN : 0759-6340

\section{Référence électronique}

Jean-François Métral, Paul Olry, Marie David, Fanny Chrétien, Philippe Prévost, Nadia Cancian, Nathalie Frère et Laurence Simonneaux, «Ruptures ou ajustements provoqués entre pratiques agricoles et enseignement de ces pratiques », Formation emploi [En ligne], 135 | Juillet-Septembre 2016, mis en ligne le 12 octobre 2018, consulté le 30 octobre 2020. URL : http:// journals.openedition.org/formationemploi/4856 ; DOI : https://doi.org/10.4000/formationemploi.4856 


\title{
Ruptures ou ajustements provoqués entre pratiques agricoles et enseignement de ces pratiques
}

\author{
Implantation et gouvernance de la réforme \\ «Produire autrement »
}

\begin{abstract}
Jean-François Métral et Paul Olry Université Bourgogne Franche-Comté, Unité propre Développement professionnel et formation, AgroSup Dijon, Didactique professionnelle
\end{abstract}

MARIE DAVID Université Bourgogne Franche-Comté, Unité propre Développement professionnel et formation, AgroSup Dijon, Sociologie

Fanny Chrétien et Philippe Prévost Université Bourgogne Franche-Comté, Unité propre Développement professionnel et formation, AgroSup Dijon, Didactique de l'agronomie

Nadia Cancian, Nathalie Frère et Laurence Simonneaux Université Jean-Jaurès, Ecole nationale de formation agronomique, Toulouse, Didactique des questions socialement vives

Résumé

Ruptures ou ajustements provoqués entre pratiques agricoles et enseignement de ces pratiques - Implantation et gouvernance de la réforme «Produire autrement »

La politique de réduction des intrants phytosanitaires (pesticides) touche les agriculteurs, mais aussi les enseignants des lycées agricoles. Ces derniers sont confrontés à des controverses, notamment au sein des classes, parmi les enfants d'agriculteurs. Nous nous centrons sur les apprentissages relatifs à la réduction d'intrants. Mobilisant une recherche pluridisciplinaire (agronomie, didactique, sociologie), nous interrogeons les structures, la division des tâches, la coordination des acteurs dans la redéfinition/appropriation de cette politique. Nous nous intéressons aux ruptures ou aux ajustements progressifs des pratiques et des normes vécus par les enseignants, les tuteurs et leurs élèves.

Mots clés : agriculture, écologie, conduite du changement, enseignement agriculture, contenu de formation, socialisation professionnelle 
Ruptures or adjustments between agricultural practices and teaching these practices - Implementation and governance of the political reform "Alternatives agricultural Productions"

Pest reduction management policy affects farmers, but also teachers of agricultural schools. They face controversial, particularly in classrooms, among the children of farmers. We focus here on learning related to the aim of the reduction of inputs, as learning object, and the debates as a learning process. Mobilizing multidisciplinary research (agronomy, teaching, sociology), this paper focus on three questions : the controversial debates as a learning opportunity; the effects of the structures, the division of labor, the coordination of actors in redefining / appropriation by the actors of the vocational agricultural training system; the interaction "on the plot" beetween farmers/student as a conceptualisation moment. We are interested in ruptures or gradual adjustments of standards and practices experienced by teachers, tutors and students.

Keywords: agriculture, ecology, change management, education by the ministry of agriculture, training content, professional socialization

Journal of Economic Literature: Q 16, I 21

Traduction : Auteur-e-s

Les préoccupations environnementales exprimées par la société interrogent les pratiques de métiers propres à l'agriculture. Le ministère français de l'Agriculture, de l'Agroalimentaire et de la Forêt les a traduites dans le projet agro-écologique pour la France'. Il définit ainsi une politique agricole du "produire autrement ». Celle-ci vise à orienter la production agricole vers une triple performance, économique, sociale et environnementale, impactant les plans institutionnel, organisationnel et socioprofessionnel. Cet article s'intéresse à la mise en œuvre de cette politique dans les situations de formation vécues par les élèves de l'enseignement agricole. Si changement il y a dans une pensée agro-écologique, comment le qualifier ? Comment les dispositifs s'en saisissent-ils ? Quelles traces en retrouve-t-on dans l'activité des acteurs des formations?

Dans une première partie, nous analysons comment le contexte de l'enseignement est potentiellement porteur de ruptures à travers l'évolution des conflits d'intérêts, des controverses professionnelles, sociales, voire familiales, concernant l'usage des produits phytosanitaires. Dans une deuxième partie, nous présentons notre méthodologie de recueil et d'analyse des données. Enfin, dans une troisième partie, nous étudions trois situations au sein du lycée : en classe, pour des enseignants d'agronomie, «discipline cour» (Martinand,

1. Puis dans la loi d'avenir pour l'agriculture du 11 septembre 2014. 
1994, p. 71) de la filière de formation des futurs agriculteurs; dans l'exploitation agricole des établissements, pour leurs directeurs ; dans les périodes en entreprise, constitutives des dispositifs de formation. En conclusion, nous esquissons quelques réflexions pour clarifier la nature de cette transition imposée, dans les pratiques, par l'injonction politique à " enseigner autrement " pour " produire autrement ».

\section{Le système d'enseignement comme levier de changement vers la transition agro-écologique}

Dans cette période de promesse de "transition vers l'agro-écologie ", cette politique convoque le système d'enseignement comme levier de changement. Ainsi, le rapport "Concertation sur l'enseignement agricole préparatoire à la loi d'avenir sur l'agriculture " (Nallet, 2013, p. 2) dresse la perspective d'une formation professionnelle préparant chaque producteur à "maîtriser par [lui-même] les combinatoires diverses et changeantes, afin de produire autrement" dans "le territoire où il se propose de vivre et dans la ou les productions qu'il a choisies». Il postule que cette perspective serait "assez généralement partagée par les responsables professionnels, les techniciens et les dirigeants politiques, parfois sous des vocables différents (...)".

\subsection{Une source politique des objets d'enseignement}

Sur ce constat d'un possible " produire autrement ", une invitation à « enseigner autrement " est lancée. Elle s'applique à tous les acteurs. Elle s'exprime en injonctions aux différents échelons de ce système d'enseignement : rénovation des référentiels de formation, collaborations attendues entre recherche-enseignement supérieur et enseignement technique, formation des personnels enseignants concernés. Elle esquisse des pistes : la nécessité de collaborer entre formateurs et professionnels, la promotion des exploitations internes aux établissements de formation; l'autonomie des établissements et leur intégration à leur territoire. Elle postule que l'ouverture sur le monde professionnel et les réseaux professionnels «fera ressource » pour mettre en œuvre les changements. Dès lors, au sein des établissements d'enseignement agricole, cette volonté politique peut conduire à infléchir, approfondir et, dans certains cas, à modifier des contenus, des pratiques et des organisations.

Notre hypothèse est la suivante : le "produire autrement " constitue une mise en cause de la rationalité technico-économique dominante concrétisée par une forte consommation d'intrants. Cette rationalité et ses principes ont fortement structuré le monde agricole, tant en termes de conception de l'action collective qu'en termes d'organisation et de normes de contrôle ( $c f$. la renégociation de la $\left.\mathrm{PAC}^{2}\right)$. Ces normes ont habitué les

2. La Politique Agricole Commune (PAC) de l'Union européenne instaure la mise en place d'aides condi- 
agriculteurs " à prendre leurs précautions » en achetant, en janvier, des phytosanitaires et des pesticides, en prévision d'un usage au printemps "puisqu'ils les ont sous la main " (un agriculteur tuteur). Ce comportement prudentiel agit comme un renforcement de la rationalité. Il témoigne d'un contrôle des pratiques porté par les services d'accompagnement des agriculteurs.

\subsection{Des controverses sociétales qui génèrent des ruptures conceptuelles, socio-épistémiques et dans les pratiques}

La forte réduction de pesticides ${ }^{3}$ espérée conduit à un changement de paradigme dans les stratégies de protection des cultures (Aubertot et al., 2005). Entendue comme objet d'enseignement, "elle génère de nombreux débats, sans espoir de solution simple et consensuelle (...) La réduction de l'emploi des pesticides est une question technoscientifique environnementale socialement vive aux plans : sociétal, éducatif, didactique " (Cancian et Simonneaux, 2013, p. 3). Pour l'enseignement, l'enjeu repose sur le paradoxe de devoir enseigner des savoirs de référence, alors même que les contenus d'enseignement à aborder sont instables et doivent pourtant être présentés comme des "savoirs à enseigner ». Il s'agit de faire des controverses passées des objets d'enseignement, sous condition d'une analyse socio-épistémologique des savoirs qu'elles mobilisent (Legardez et Simonneaux, 2006). Avec les élèves sont retracés, d'une part, la nature des ruptures et des ajustements relatifs aux contenus à enseigner et, d'autre part, les arbitrages rendus, les compromis passés entre acteurs de l'enseignement agricole sur le savoir à enseigner. Deux moments sont à retenir.

Le premier concerne le début des années 1980, où les coopératives agricoles, les chambres d'agriculture et l'Union des industries de protection des plantes (UIPP) promeuvent la "parcelle zéro défaut ", à l'attention des agriculteurs intensifs ; pour ces derniers, la parcelle de culture devient la vitrine de leur compétence et de leur réussite. Les centres de recherche agronomique participent à la mise au point d'innovations, instruisent des protocoles et conseillent les décideurs : le modèle est la plante saine. Des alliances entre une partie de la recherche agronomique, les agriculteurs " conventionnels ", les firmes et l'UIPP diffusent un discours technique standardisé (déqualifiant l'agronomie) sur les menaces que font peser les bio-agresseurs et sur le confort généré par le recours aux pesticides. La mise en cause de l'innocuité des pesticides est " neutralisée » par des contrôles des pesticides comparables à ceux effectués pour les médicaments. Ainsi les firmes, la structure de contrôle des pesticides, une recherche agronomique analytique plutôt techno-centrée,

tionnées par le respect de règles en matière d'environnement et de bonnes conditions agricoles et environnementales (BCAE) et des contrôles correspondants. Ces contrôles portent par exemple sur le stockage des produits phytosanitaires, l'enregistrement de toute utilisation de ces produits, le respect des limites maximales de résidus de pesticides dans les produits récoltés...

3. Nous employons ici le terme " pesticides » au sens de produits phytosanitaires, dont il est espéré une diminution de $50 \%$ d'ici 2025, dans le cadre du plan Ecophyto 2018. 
et la FNSEA ${ }^{4}$ s'opposent à une recherche agronomique d'approche systémique portée par des spécialistes de la santé et des écologistes, dont les arguments ne diffusent pas dans la sphère publique.

Le second moment concerne la fin des années 2000, où les pesticides sont reconnus «substances dangereuses" (directive 2009/128/Communauté européenne) dont il faut limiter les usages. S'il y a consensus sur les effets des pesticides sur l'eau et les sols, les débats restent vifs au sujet des abeilles et de la santé des utilisateurs. Le Grenelle de l'Environnement ${ }^{5}$, les expertises sur les alternatives et des rapports convergents sur les pesticides jouent un rôle-clé dans les controverses. Les discours sur la "santé des consommateurs " d'abord, puis sur celle des utilisateurs, structurent l'argumentation en faveur de la limitation, voire de la suppression des pesticides de synthèse. Ils pointent les incertitudes liées aux effets de ces pesticides, particulièrement à long terme. Les controverses se déploient donc vers des fronts mobilisateurs, les effets sur l'environnement biophysique restant en arrière-plan. Les uns prônent une utilisation raisonnée et durable des pesticides, garante d'un niveau élevé de production pour assurer les besoins alimentaires liés aux prévisions démographiques (UIPP, FNSEA). Les autres, agriculteurs et chercheurs ayant développé des systèmes alternatifs aux pesticides, coproduisent des savoirs illustrant un nouveau rapport aux savoirs et au contexte. Pour eux, l'intensification des processus écologiques sera garante de durabilité (promoteurs de l'agriculture écologiquement intensive, dont l'INRA ${ }^{6}$ et des agriculteurs alternatifs).

La répétition des crises sanitaires, entre 1983 et 2007, génère une défiance croissante à l'égard des industries chimiques et des implications des activités scientifiques. La diversité des thématiques, la dispersion des arguments, l'absence de front d'opposition net, constituent un empêchement pour les enseignants dans la construction d'un enseignement tenu par des références. A partir de 2007, grâce à leur capacité à recroiser des informations, à imposer la mise à disposition de rapports d'études scientifiques et à communiquer, des alternatifs et/ou des opposants aux pesticides deviennent des producteurs de savoirs. Les pesticides sont assimilés à des substances dangereuses dont il faut limiter les usages. Dès lors, le travail des enseignants se cristallise sur le « comment » enseigner aux futurs exploitants d'autres façons de faire que celles de leurs parents.

Le plan Ecophyto 2018 y apporte un sens qui clôt les controverses, avec l'objectif de limiter, à échéance de dix ans, l'emploi des pesticides $(50 \%)$. La réduction de l'usage des pesticides entraîne des ruptures conceptuelles (façons de penser), socio-épistémiques (façons de comprendre) et pratiques (façons de faire), mettant le champ conceptuel agronomique sur le devant de la scène. Ces ruptures constituent autant de situations d'enseignement-

4. Fédération nationale des syndicats d'exploitants agricoles.

5. Lancé en juillet 2007, le "Grenelle de l'Environnement " a réuni les représentants de l'Etat et de la société civile pour prendre des mesures à long terme en faveur de l'environnement et du développement durable. 6. Institut national de la recherche agronomique. 
apprentissage rencontrées par les acteurs de l'enseignement agricole, et en particulier les élèves, du fait des relations directes ou indirectes qu'ils entretiennent avec d'autres acteurs du monde agricole (leurs pairs, leurs familles, les professionnels intervenant dans la formation...). Comme le signalent Cancian et Simonneaux : "Les acteurs de la situation didactique, enseignants et élèves, ne peuvent échapper aux débats polémiques (...), reflets de controverses sociétales largement médiatisées. " (2013, p. 3)

Ce tableau initial, bien incomplet, fonde notre contribution au présent dossier : l'appropriation, par les acteurs, de cette politique publique, conduit-elle à une rupture avec des pratiques et des normes antérieures dans l'enseignement et/ou à des ajustements progressifs? Nous n'abordons pas la question en politistes, mais sous l'angle des représentations des acteurs.

\section{Apprendre des contenus techniques et professionnels instables : méthodologie d'une recherche pluridisciplinaire}

Pour répondre aux questions qui précèdent, nous mobilisons une recherche en cours ${ }^{7}$. Elle vise à identifier les contenus et les situations d'enseignement-apprentissage favorisant une évolution des pratiques agricoles économes en produits phytosanitaires. Les actions engagées s'inscrivent dans des cadres théoriques de différentes didactiques (didactique des questions socialement vives (QSV), didactique disciplinaire, didactique professionnelle).

Nous présentons tout d'abord l'approche conceptuelle globale qui fonde notre méthodologie de recueil et d'analyse des données. Nous décrivons ensuite les données recueillies et l'articulation pluridisciplinaire de la méthodologie utilisée.

\subsection{Fondements conceptuels globaux de la méthodologie}

Les représentations des acteurs sont susceptibles de ruptures conceptuelles vis-à-vis des modèles que le "produire autrement " convoque. Ainsi, le concept de système de culture (Sebillotte, 1990) est constitué en cadre de l'action des agriculteurs en production végétale. Il appelle à un changement d'échelle pour les exploitations (ne plus penser les parcelles une à une mais en système) et pour les objets que ce système doit prendre en compte (eau, biodiversité, etc.).

Ces ruptures peuvent aussi être "socio-épistémiques ». L'élargissement des préoccupations aux trois " piliers » du " produire autrement » suscite en effet des débats concer-

7. En réponse à l'appel à projet " Pesticides ", cette recherche « Didacphyto ", lauréate du programme Pesticides 2013, est financée par l'Office national de l'eau et des milieux aquatiques (plan Ecophyto 2018). 
nant des orientations sociopolitiques mais aussi des dimensions techniques, relevant autant du scientifique que d'un rapport à la pratique (Bourdieu, 1980).

Enfin, ces ruptures peuvent être " pragmatiques ", ayant trait tout autant aux raisonnements qu'aux conceptualisations émergentes des professionnels face aux conséquences des évolutions. Ainsi, par exemple, l'idée des conséquences des pesticides sur la santé progresse, conduisant à une pratique «conscientisée » (DARES, 2003 ; Molinié, 2010).

Dès lors, les acteurs des terrains éducatif et productif doivent trouver les termes d'un accord qui n'est pas formel, mais se construit sur deux plans : d'une part, les significations que chacun élabore sur le "produire autrement» (Simonneaux, 2011, 2013); d'autre part, l'action qui en découle pour faire connaître, comprendre, agir autrui (Mayen, 2007). Il s'agit de passer d'une injonction politique à rompre avec les pratiques agricoles antérieures, à l'objectif d'instruire et de qualifier, avec les élèves, le saut de raisonnement, la rupture de pensée et le changement de pratiques attendus qu'elle suppose.

Notre regard se construit donc d'abord par l'étude des controverses qui déstabilisent un contenu d'enseignement. Les savoirs agronomiques de base ne sont pas mis en défaut. Ils sont au contraire mis en valeur par la démonstration de validité des alternatives agroécologiques ; en effet, cette démonstration implique l'explication, auprès des élèves, des effets de la combinatoire des techniques, dans un empan temporel élargi à la rotation et à la mobilisation des services agro-systémiques. Ainsi par exemple, le concept de système de culture constitue un défi en matière d'enseignement et d'apprentissage : comment expliquer, comment comprendre ce système désigné comme un ensemble de parcelles cultivées de façon homogène, qui se caractérise par (i) la nature des cultures et leur ordre de succession (la rotation des cultures), (ii) les itinéraires techniques appliqués à ces différentes cultures (ensemble des opérations effectuées sur un cycle cultural), ce qui inclut le choix des variétés pour les cultures retenues (Sebillotte, 1990). Comment et jusqu'où expliciter une telle pensée systémique ordonnée sur le vivant, à des élèves de seconde, de bac pro et de BTS (brevet de technicien supérieur) ? Comment le faire lorsque les savoirs de la tâche (Savoyant, 2008) ne peuvent ni être formalisés, ni faire l'objet de descriptions objectives prescriptes concernant les systèmes et les processus ? Comment enseigner ces savoirs d'ordre technologique se présentant comme hétérogènes, complexes, mais devant être enseignés?

Ajoutons que nous parlons ici de formation préparant à une profession, inscrite dans des espaces sociotechniques, mobilisant des savoirs de l'activité qui orientent la réalisation effective en contexte situé, impliqués au double plan de la réussite immédiate et de l'efficience à long terme. Ces savoirs de l'activité relèveraient, selon Savoyant (op.cit.), d'une intelligence pragmatique qui mobilise des savoirs d'ordre proprement professionnel, dont l'appropriation requiert l'exercice en milieu de travail. Ces savoirs de l'activité, toujours singuliers, sont incorporés par les exploitants agricoles. Comment, lors des tutorats par exemple, peut-on y avoir accès ? Sous quelle forme? Nous recourons ici à la notion de "représentations pour l'action" (Teiger, 1993), en tant que réseau de connaissances, de 
savoirs et de savoir-faire, sélectionné au titre de ce que l'action exige. Leur fonctionnalité réside dans le guidage de l'action organisée pour l'atteinte de son but. Fonctionnelles, ces représentations sont caractérisées par "les propriétés de leur contenu et les modalités organisatrices de l'activité qu'elles autorisent " (Teiger et Laville, 1989, p. 56). Ainsi, plusieurs agriculteurs se distingueront par la représentation plus ou moins précise qu'ils ont du sol comme structure et comme texture, chaque façon de le penser organisant différemment la pratique.

L'enjeu professionnel de l'agro-écologie porte ainsi sur l'apprentissage des savoirs instables et des conditions qui le permettent, au sein de triades enseignants / tuteurs / élèves, en lien aux situations qui les mobilisent.

\subsection{Méthodologie : une recherche pluridisciplinaire en trois phases}

Nous traitons les questions posées plus haut au travers du contenu et des modalités d'enseignement pour la formation au baccalauréat professionnel CGEA SDC ${ }^{8}$, pour les situations d'enseignement en classe et les situations de formation en exploitation agricole.

Nous centrons nos travaux sur la thématique de la réduction de l'usage des produits phytosanitaires dans la tâche de gestion des adventices des cultures (" mauvaises " herbes). Nous le justifions, tout d'abord, par le fait que cette thématique est emblématique de la problématique du " produire autrement » et des questions qu'elle soulève pour la formation des futurs agriculteurs ; cette tâche présente l'intérêt d'intégrer toutes les dimensions de la complexité de la gestion du vivant au sein de l'agroécosystème. Nous le justifions, ensuite, par le fait que ce niveau de diplôme concrétise cet objectif sur le plan socioprofessionnel, puisqu'il peut permettre aux futurs agriculteurs d'obtenir les aides à l'installation.

Notre méthodologie s'articule en trois phases :

1 - Repérage de ce qui fonde le rapport à l'usage des produits phytosanitaires dans les interactions élèves/maître de stage : les controverses (voir $\$ 1$.) :

En référence au cadre de la didactique des questions socialement vives, l'analyse ${ }^{9}$ diachronique relative à l'usage controversé des pesticides prend appui sur une enquête socio-épistémologique qui dresse un état à la fois des savoirs de référence et des éléments du contexte de leur émergence. Le corpus de références a été construit à partir d'une revue bibliogra-

8. Conduite et Gestion de l'Exploitation Agricole - Systèmes à Dominante Cultures.

9. En référence à la Théorie de l'Acteur-Réseau (ANT) (Callon, 1986 ; Latour, 1989; 2007) et la théorie des biais culturels de Douglas \& Wildavsky (1983). L'ANT s'intéresse à la dynamique et à la circulation des savoirs, en particulier au cours des processus de "traduction ", selon la terminologie de Latour (processus qui permettent la compréhension de l'énoncé initial par un tiers). Elle propose de voir comment les acteurs construisent leur monde, comment ils le définissent, comment ils s'associent, ils négocient et se disputent à propos d'un monde qu'ils souhaiteraient : il s'agit de décrire et d'analyser leur monde en train de se faire et en particulier les controverses qui les opposent. 
phique internationale, en veillant à capter la pluralité des points de vue et à intégrer le caractère distribué des savoirs (cent sources, avec des publications primaires et secondaires variées). Le recours à la controverse vise à développer, chez les élèves, des capacités d'enquête et d'argumentation inhérentes à la capacité de décision. De ce point de vue, des cartographies rendent compte des reconfigurations d'alliances, des renforcements de position, de la variation dans la justification des arguments de chaque actant (sphère politique et réglementaire, les mondes professionnels agricoles - firmes phytosanitaires, organisations professionnelles agricoles, agriculteurs, monde scientifique et du développement).

2 - Analyse de la multi-référentialité des savoirs chez des enseignants, des directeurs d'exploitations agricoles de lycée ${ }^{10}$ et des agriculteurs maîtres de stage ou d'apprentissage :

Deux études de cas de baccalauréat professionnel CGEA dans deux lycées en action 16 du plan Ecophyto ${ }^{11}$ ont permis d'analyser les logiques et les configurations didactiques (Simonneaux, 2011) mises en œuvre par des enseignants. Des entretiens semi-directifs relatifs à leurs pratiques didactiques et pédagogiques ont été conduits auprès d'eux, puis de leurs élèves, sur la base d'une "question d'évocation " (cf. Approche structurale des représentations sociales; Abric, 1994). Ces données se complètent d'observations en classe de séquences d'exploitation du stage (écriture de " fiches d'activité ", écriture du rapport de stage, préparation à l'épreuve professionnelle du baccalauréat). Réciproquement, les savoirs d'action (opératifs selon eux) pour la réduction des pesticides sont enquêtés par des entretiens semi-directifs menés auprès de quinze agriculteurs (trois directeurs d'exploitations agricoles de lycées et douze agriculteurs faisant partie du réseau des maitres de stages de ces lycées).

Nous étudions ici, dans le discours des enseignants et des agriculteurs, l'intégration de la multi-référentialité des savoirs qui est proposée aux élèves aux fins de mobilisation dans l'action.

3 - Analyse des représentations des professionnels encadrant les apprenants lors des mises en situation professionnelle :

Dans ce contexte d'évolution des pratiques agricoles vers la réduction d'intrants, nous procédons enfin à l'analyse des apprentissages d'élèves en stage dans des exploitations agricoles par :

- des questionnaires et entretiens collectifs en retour de stage ;

10. Chaque Etablissement public local d'enseignement et de formation professionnelle agricole comporte une exploitation agricole en tant que centre constitutif de l'établissement.

11. L'action 16 du plan Ecophyto vise à «engager les exploitations de l'enseignement et du développement agricoles à jouer systématiquement un rôle moteur dans la généralisation des itinéraires techniques et des systèmes de culture innovants", notamment à travers les méthodes "économes en pesticides» et la participation au réseau d'expérimentation / démonstration (Comité national d'orientation et de suivi Ecophyto 2018 - 22 avril 2009 http://agriculture.gouv.fr/IMG/pdf/ecophyto2018-avril09.pdf). 
- des entretiens à différentes étapes du parcours avec 26 élèves/apprentis de classes de 1 ìre et terminale de Bac Pro CGEA (et avec leurs parents pour les enfants d'agriculteurs), avec leurs maîtres de stage ou d'apprentissage (MS/MA);

- des entretiens initiés par nous sur le lieu de stage entre le MS/MA et les apprenants concernant le système d'exploitation.

L'objectif est ici, sur la base de traces filmées d'échanges en " bout de champ ", de saisir les raisonnements déployés en situation de travail par les élèves, en référence aux capacités travaillées au lycée par leurs enseignants, aux pratiques transmises par leurs maîtres de stage ou/et par leurs parents.

\section{Apprendre à produire autrement : entre ruptures 3 et ajustements}

Nous nous appuyons sur cette méthodologie pour envisager les conditions d'un enseignement contributif à la politique de réduction de l'usage des produits phytosanitaires et donc du "produire autrement». Nous examinons ici différentes situations participant à la formation des futurs agriculteurs : situations d'enseignement en classe ; situations de formation dans ou à propos de l'exploitation du lycée ; situations de formation en exploitation agricole hors lycée.

Nous examinons en quoi ces situations révèlent des ruptures et/ou des ajustements progressifs dans les représentations concernant l'usage des produits phytosanitaires et son enseignement, successivement chez des enseignants, puis chez des agriculteurs, dont des directeurs d'exploitations agricoles de lycée, et enfin chez des élèves et leurs maîtres de stage.

\subsection{La réduction des phytosanitaires, objet risqué à apprendre comme à enseigner}

Une rénovation récente des curricula de l'enseignement agricole intègre les recommandations du plan Ecophyto 2018. Elle pointe un nécessaire changement d'approche dans la protection des cultures, susceptible de produire des ruptures conceptuelles, socio-épistémiques et pragmatiques se manifestant, en classe, pour les enseignants d'agronomie et leurs élèves.

Tout d'abord, en classe, les logiques et les configurations didactiques (Simonneaux, 2011) des enseignants enquêtés présentent un double objectif commun :

- former les élèves au raisonnement, à l'argumentation ;

- savoir identifier les objectifs et la stratégie des agriculteurs en observant leurs pratiques. 
Deux enseignants ont retenu notre attention au regard d'une même situation prescrite d'enseignement (la réduction d'intrants sur une parcelle de culture en blé d'hiver) qu'ils décrivent par des caractéristiques différentes. En effet, le risque majeur à prévenir n'est pas le même pour l'un et l'autre, selon un processus de légitimation qui ne vise pas les mêmes acteurs.

Pour l'un, ce thème porte un risque d'enseigner majeur, qu'il convient de prévenir, au regard des tests réalisés sur l'exploitation du lycée. Il perçoit les professionnels comme aux aguets de toute baisse de rendement sur les parcelles expérimentales du lycée. Il ne tire plus la satisfaction attendue de son investissement dans la recherche expérimentale d'alternatives à l'usage intensif de produits phytosanitaires au sein de l'exploitation agricole du lycée. Il est inquiet de la propension des élèves à diffuser, dans leurs environnements, des messages déformés en défaveur de l'image de l'exploitation du lycée. Il doit reconstruire une légitimité auprès des acteurs sociaux les plus sceptiques, en faisant appel à ceux qu'il appelle « les pairs" : les agriculteurs innovants qui découvrent des « astuces » pour réduire leur consommation en pesticides. Comme outil pédagogique, il privilégie l'étude de cas en situation réelle, chez des agriculteurs, plus ou moins en substitution à l'observation des parcelles d'expérimentation du lycée, sur lesquelles les résultats ne sont pas toujours visibles. En mixant les savoirs et leurs applications, il se met à l'abri de reproches redoutés. Dans son enseignement, il s'applique à modérer les débats concernant la question de la réduction de l'usage des pesticides, question vive pour des enfants d'agriculteurs, et de surcroît exigeante en termes de construction d'un raisonnement critique des pratiques d'aujourd'hui, au nom d'une certaine vision de l'agriculture de demain.

Pour l'autre enseignant, les situations d'enseignement qu'il pourrait proposer sont trop éloignées des pratiques sociales et professionnelles de référence de ses élèves. Egalement convaincu des effets néfastes des produits phytosanitaires sur le diptyque santé/environnement et de l'ignorance de ses élèves à ce propos, il opte pour une stratégie qui priorise la prise de conscience de ces deux dangers. Face aux élèves, le thème " réduire l'usage des pesticides " organise l'enseignement selon une configuration critique et un discours alarmiste. En donnant ses points de vue, il ouvre vers de possibles débats, au cours desquels les élèves vont devoir argumenter. Il les prépare ainsi à sortir de leur contexte, à prendre du recul, à "s'ouvrir l'esprit ». Afin de légitimer cette posture sceptique et une rationalité critique et sociale, il s'appuie sur les savoirs de référence du nouveau référentiel et articule ses enseignements avec de nombreux intervenants extérieurs, notamment des professionnels du développement agricole et de la santé. Ces témoignages, situés, constituent de véritables savoirs contextualisés qui prennent sens par rapport aux représentations des élèves.

Les deux enseignants enquêtés sont ainsi conduits à "refroidir " ou à " réchauffer ", pour leurs élèves (Simonneaux, 2013), cette question vive de la réduction de l'usage des pesticides et phytosanitaires, par la mise en débat des représentations, des points de vue 
d'élèves, d'agriculteurs et de parents d'élèves locaux. Ils considèrent que leur légitimité dépend de celle qui leur est accordée par les agriculteurs.

Ces deux cas de figure mobilisent des ajustements conceptuels et socio-épistémiques, des débats sur les pratiques, sur les savoirs de la tâche, que les controverses entre acteurs alimentent.

Du point de vue des élèves, les enseignants travaillent la capacité à se saisir d'éléments de connaissances perturbées et perturbantes, au regard des pratiques et des techniques. Par exemple, les rotations de culture sont travaillées en classe, en soulignant l'importance de les penser en référence à l'exploitation, au territoire (changement d'échelle). Cette façon de penser systémique est soutenue par des apports conceptuels et un traitement des impasses et obstacles de compréhension révélé par l'étude des controverses. Comment agir en tant que futur exploitant sur des bases conceptuellement construites, argumentées ? Cette interrogation constitue un des enjeux de cet enseignement progressivement ajusté pour contenir les tensions entre finalité éducative et finalité productive qu'il induit.

\subsection{Représentations pour l'action : un débat socio-professionnel sur les normes de pratiques}

Le plan national «Enseigner à produire autrement " met l'exploitation agricole des lycées au cour des enjeux de formation des futurs acteurs professionnels. Elle doit " devenir un exemple pour les professionnels, un démonstrateur des transitions vers des systèmes novateurs et durables, une tête de réseau pour le développement local " et "contribuer à la transmission de valeurs et de comportements des futurs professionnels qui sont un élément essentiel dans la construction de l'identité professionnelle des futurs agriculteurs et des futurs citoyens qu'elle forme " (Prévost, 2013, p. 155).

Pour cela, toute exploitation agricole de lycée doit viser une double ambition : un système de production agro-écologique certifié et une utilisation pédagogique intensifiée ${ }^{12}$. De ce fait, les directeurs de ces exploitations doivent tenir compte des représentations et pratiques des acteurs (agriculteurs, services d'appui et de développement, réseaux d'innovation...) de leur territoire. Souvent, ces derniers interrogent la transition agro-écologique, voire s'y opposent dans le discours, tout en étant actifs au sein de leur exploitation :

" Je n'ai jamais vu la vie biologique tuer les mauvaises herbes moi hein! Ou alors, j'ai pas dî regarder la bonne vie... Il faudra expliquer aux prêles, aux gaillets et à la folle avoine que maintenant, il faudrait qu'ils aillent ailleurs, mais à mon avis, ils vont pas trop écouter!" (...)

12. Note de service «Mobilisation pour la transition agro-écologique des exploitations agricoles et des ateliers technologiques de l'enseignement agricole » - DGER/SDRICI/2015-138, du 16 février 2015, de la Direction générale de l'enseignement et de la recherche du ministère de l'Agriculture. 
"Non, mais on est tout de même en train de bosser là-dessus. Moi, je regarde un peu ce qui se fait... Je pense que je fais un peu partie des avant-gardistes, je me suis équipé d'un strip-tilll'3 depuis trois ans, jai des expériences de tentatives de couverts végétaux, donc jai un peu regardé quand même, je ne parle pas sans savoir. Mais ça me parait pas du tout évident!" (Un agriculteur maitre de stage et proche d'un lycée agricole de Midi-Pyrénées)

Ces points de vue paradoxaux conduisent les directeurs d'exploitation à assumer un rôle de médiateur, entre la compréhension du point de vue du professionnel et le besoin d'élargir les enjeux de la réduction dans l'usage des produits phytosanitaires, quand bien même ils considèrent que les agriculteurs ont abandonné la vision globale de leur rôle :

"Je vais être un peu violent dans mon discours mais (...), la vraie expérience d'agronome, on ne la trouve quasiment plus que chez les agriculteurs bio. Les autres ont perdu la notion de l'impact de leurs pratiques sur le milieu qui les entoure. " (Un directeur d'exploitation de lycée agricole de la région Centre)

Ces extraits nous révèlent à la fois les représentations du "produire autrement ", et la manière dont se déclinent les niveaux de problèmes auxquels se heurte l'enseignement du " moins d'intrants " : un obstacle normatif (la vie biologique est envahissante), une tension axiologique (valuation du moins d'intrants), des impasses épistémiques (l'expérience des traitements $v s$ les alternatives agronomiques).

De ce point de vue, les deux extraits du discours du maitre de stage constituent des indices de telles ruptures et d'ajustements progressifs sur les plans conceptuel et pragmatique. De plus, les mots et justifications utilisés par ces deux acteurs révèlent des écarts de représentation et de rapport au savoir autour de l'usage des produits phytosanitaires. Le discours du maître de stage utilise principalement des arguments issus de sa propre expérience et centrés sur la technique : les "manvaises herbes" et les moyens techniques pour les "tuer» (strip-till, couverts végétaux). Le discours du directeur d'exploitation se réfère à des connaissances de l'agronome (voire de l'agronomie), élargissant la situation à «l'impact de leur pratique sur le milieu qui les entoure".

Ces propos questionnent les possibilités de construction d'un accord formel ou informel concernant les représentations et significations du " moins d'intrants ", entre le directeur et les autres acteurs de son territoire (agriculteurs, services d'appui et de développement, réseaux d'innovation...). En creux, se trouve ainsi interrogé le rôle assigné aux exploitations agricoles des lycées dans le cadre du plan national « Enseigner à produire autrement ». Mais ce rôle de modèle, convoquant un choix de systèmes de culture et

13. Le strip-till est une technique de travail du sol où seul le futur rang de semis est travaillé, laissant les résidus des cultures précédentes en surface dans l'inter-rang : l'enjeu est la non-perturbation du sol, la conservation de l'humidité et la réduction des levées adventices. Techniquement, le matériel ne laboure pas : il prépare la terre en nettoyant la ligne de semis, en la fissurant, en mélangeant les débris végétaux à la terre, ce qui créé de la terre fine pour un lit de semences. 
d'expérimentations mises en place sous le regard des autres acteurs du territoire, se veut d'abord une affirmation de pratiques re-normalisées.

Pour autant, dans le cadre de la mission des exploitations de lycée, ces pratiques re-normalisées supposent moins un dépassement des obstacles, impasses, tensions, qu'une implantation d'une façon de faire économe en intrants en termes de possibilité, faisabilité, efficience des pratiques. Pour le directeur, cette façon de faire implique d'expliciter la réduction de l'usage des produits phytosanitaires imposée de l'extérieur, en lien avec les acteurs du territoire dans lequel s'insère l'établissement.

Cela relativise les représentations d'un changement rationnellement conduit au sein du cadre socio-organisationnel d'un établissement. L'enjeu du changement tient plus en effet aux ajustements conceptuels, socio-épistémiques et pragmatiques. Ces derniers viennent doubler la transition organisationnelle de l'établissement imposée par la politique de l'enseignement à " produire autrement ", en réduisant l'usage d'intrants chimiques.

Finalement, cette politique invite à une transition. Il s'agit de faire reposer l'enseignement non sur des savoirs instables, mais sur la mise en débat, la mise sous expérimentation de représentations pour l'action. Ce glissement des références pour enseigner suppose, pour les acteurs de l'établissement (enseignants, directeur et techniciens de l'exploitation), de :

- se coordonner et trouver un accord sur des équivalences entre savoirs scientifiques et savoirs techniques ;

- se situer par rapport à " ce qu'ils tiennent pour vrai » (et non au regard d'une vérité scientifique) pour justifier leur action au regard de la situation ;

- se reconnaître une légitimité à garantir une conformité aux règles de métier.

\subsection{Les mises en situations professionnelles : espaces contradictoires de socialisation des futurs agriculteurs}

Les ruptures et ajustements évoqués ci-dessus se concrétisent dans les espaces de socialisation que constituent les moments passés par les élèves ${ }^{14}$ et 15 dans des exploitations extérieures à l'établissement : périodes de stage pour la voie scolaire et périodes en entreprise

14. Afin de faciliter la lecture, nous emploierons le terme de stagiaire ou d'élève sans distinguer s'il s'agit d'élève suivant la formation par la voie scolaire ou par apprentissage.

15. Plus de $27 \%$ des élèves du second degré (CAPA, Baccalauréat général, technologique et professionnel) dans le secteur de la production (agronomie, agriculture, productions végétales, productions animales) sont des enfants d'agriculteurs ou d'ouvriers agricoles (Direction générale de l'enseignement et de la recherche : DGER -, ministère de l'Agriculture, de l'Agroalimentaire et de la Forêt (2012). Panorama de l'enseignement agricole). Comme le montre notre enquête, il faut y ajouter les nombreux élèves d'autres origines socioprofessionnelles qui participent aux activités d'exploitations agricoles d'un cercle familiale élargi (oncles, cousins, amis...) ou d'exploitations situées à proximité de leur domicile, ceci dans le cadre de jobs d'été, voire en dehors de tout cadre défini. 
pour l'alternance, constitutives du dispositif de formation ; moments de participation aux activités de l'exploitation familiale ou d'autres exploitations agricoles à proximité du domicile. A ce titre, ils participent aux différentes activités d'une exploitation agricole; ils occupent différentes places au travail (Delbos et Jorion, 1984) et se constituent un modèle de référence du " professionnel ", concernant notamment les produits phytosanitaires dans la gestion des adventices. Or, ce modèle peut s'opposer à ceux proposés dans l'enseignement du "produire autrement » et de l'agro-écologie ${ }^{16}$.

Dès lors, ces mises en situations professionnelles cristallisent le débat de normes, qui n'est pas nouveau, mais qui nous semble réactualisé par la transition agro-écologique. C'est ce qu'illustre l'extrait d'un entretien avec Luc (élève de bac pro CGEA, fils d'agriculteur) :

"Luc: Raisonner un peu ses traitements. C'est ça qui me stoppe un peu. J'ai envie d'arriver à avoir comme la herse étrillele, mais mon père il me dit: "non, il faut peut-être pas faire ça". Après voilà, je voudrais essayer, mais c'est un peu dur à lui demander de faire ça.

Enquêteur : Mais ça, il n'est pas totalement fermé sur ça?

Non. (...). Il n'est pas tout à fait d'accord et pas tout à fait en désaccord. Après voilà, on discute.

Enquêteur: Toi, ce qui támènerait là, c'est pour réduire les intrants, ce que tu disais tout à l'heure?

Luc: Les IFT $T^{18}$. Je voudrais essayer de raisonner un peu... pas complètement mettre de côté les traitements, juste en rattrapage.

Enquêteur : Et ça, tu l'as appris où?

Luc: A l'école. J'y ai réfléchi en stage.

Enquêteur: Chez ton maître de stage, Monsieur L ?

Luc : Et après, oui je l'ai appris chez moi aussi : essayer de parler avec mon père, pour réduire les IFT. "

Dans la mesure où le débat porte davantage sur le « comment il faut faire " que sur le " comment faire ", il s'inscrit dans un jeu de dépendances entre la famille, l'école et la réflexion des élèves. Nous suggérons même de parler de dettes croisées et de dépendances réciproques, car les élèves restituent, dans leurs propos, cet enjeu de trouver leur place, de se faire leur vérité dans des espaces de significations brouillés. Et ce débat ne se limite pas à la définition du " professionnel » dans les interactions de l'élève avec les

16. Ces oppositions sont bien décrites dans Lamine (2011).

17. Outil de désherbage mécanique très utilisé en agriculture biologique, en production céréalière.

18. Indice de Fréquence de Traitements : indicateur utilisé pour le suivi de l'évolution de la consommation de pesticides. 
professionnels qu'il côtoie. Il se joue aussi autour de l'objet « savoirs enseignés » dans les interactions qu'il a avec les enseignants, mais aussi dans celles entre les enseignants et les professionnels du monde agricole, voire entre les enseignants et leur institution.

Dans le rapport des savoirs agro-écologiques aux pratiques et situations professionnelles, l'enseignement d'agronomie apparaît comme un espace intermédiaire qui articule des savoirs et des pratiques - les premiers intervenant en légitimation des secondes - et au sein duquel les pratiques acceptables sont fondées sur les savoirs. De ce point de vue, les enseignants reconnaissent la multi-référentialité des savoirs. Ils recherchent l'intégration des savoirs d'origine différente, mais n'ont pas tous le même degré de maîtrise des savoirs "professionnels ", notamment en raison de leur carrière : certains ont exercé le métier auquel ils préparent les élèves et peuvent situer ces savoirs avec précision; d'autres y parviennent à force de fréquentation, depuis de longues années, du même secteur professionnel qui a permis une dynamique "d'acculturation réciproque " avec les tuteurs et les employeurs; d'autres, enfin, n'en ont qu'une représentation lointaine, à partir de leur expérience d'usager, par exemple.

A ce propos, le point de vue des stagiaires est différent. Pour eux, en effet, dans plusieurs cas, la qualification d' "agro-écologique " attribuée aux savoirs tend à les inscrire dans la sphère scolaire et à limiter leur sens et légitimité dans la sphère professionnelle (ou familiale), tout en assurant la valorisation de cette dernière. A leurs yeux, ces savoirs agro-écologiques ne sont pas toujours reliés aux savoirs, aux situations et aux pratiques professionnelles dans l'enseignement. Dans cette conception, il semble qu'il y ait deux catégories de savoirs, en fonction de leur sphère de légitimité et de pertinence. Il existe, d'une part, les savoirs scolaires de l'agro-écologie, relevant de l'enseignement, et nécessaires pour réussir aux épreuves de validation ; d'autre part, les connaissances professionnelles, relatives à la pratique en entreprise, et indispensables pour être reconnu dans les situations de travail.

Cela semble renforcé par le fait que, du côté des professionnels, l'élève peut entendre une autre version du savoir en jeu, dans les tâches à apprendre (Olry et Astier, 2001), que ce qui lui est proposé du côté scolaire. C'est le cas, par exemple, de savoir-faire relatifs à la gestion des adventices, dont la mobilisation s'opère sous la forme de conceptualisations pragmatiques du côté des professionnels (les savoirs ne sont pas mobilisés " en leur état scolaire ", mais via un système de connaissances qui engendre l'activité). Ainsi en est-il des deux termes de distribution et de couvert, qui ont une portée conceptuelle, comme l'indique l'extrait suivant :

"Maitre de stage: Maintenant, on essaie de plus jouer sur la rotation dans des terres comme ça. Comme on a l'usine de déshydratation de luzerne à côté, de faire un peu plus de luzerne sur l'assolement et de l'implanter sur des terres comme celle-ci. Bien que ce soit des terres à colza, on n'arrive plus à en faire un. Ce sont des colza qui nous coûtent très cher à produire, avec des rendements qui sont très très médiocres. (...) 
Enquêteur : et alors, c'est quoi l'intérêt de la luzerne?

Stagiaire: La luzerne, elle est bien parce qu'elle couvre le sol et du coup, elle empêche la levée des adventices et elle apporte de l'azote à la parcelle. Elle capte l'azote de l'air pour le redistribuer dans le sol. (...)

MS : Là, on est en culture de blé après une culture de luzerne. Y'a juste des pissenlits qui repoussent, mais ça c'est rien, ça se désherbe très facilement... "

Il convient ici d'entendre, dans le propos du maître de stage, la mobilisation, conceptualisée, du rôle de la luzerne introduite dans la rotation : elle capte l'azote et le redistribue dans le sol, elle étouffe les adventices et protège donc la croissance du blé à venir ; accessoirement, elle évite le coûteux désherbage mécanique de la parcelle. Pourtant, écoutons la fin de cet échange :

"Enquêteur : Et comment on fait... enfin, c'est quoi quand vous dites "ça se désherbe"?

MS : Ben, avec une application de... tiens, ben, d'ailleurs, une repousse de luzerne, ça, c'est de la luzerne qui n’a pas été détruite. (...) J'ai essayé de la détruire mécaniquement avec des outils à dents puis à disques après. Et y'avait pas mal de repousses. Et du coup, on a appliqué du glyphosate dessus pour éviter qu'elle repousse. Même avec le glyphosate, on voit que (rire) ça marche pas» (échange tutoral en bout de champ, en présence du maître de stage et son stagiaire).

C'est alors par son activité (et non par un cours sur la fertilisation azotée, sur la gestion des adventices) et dans l'échange itératif avec le tuteur et l'enseignant référent que le stagiaire se forge une représentation pour l'action. Cette émergence peut, comme ici, sembler contradictoire : le maitre de stage développe un raisonnement agro-écologique, puis semble y renoncer pour dépasser son impuissance avec du glyphosate (produit cancérogène). Le stagiaire et son tuteur alternent " recours " et " disqualification " des savoirs agro-écologiques dans l'action professionnelle. Cette façon de penser clive la façon de se former entre un savoir à apprendre, qui se communique, et des compétences construites et transformées dans l'action, sans lien entre les premiers et les secondes. De ce point de vue, la conception des élèves, attachée aux impératifs agro-écologiques n'est, tout compte fait, pas si différente de celle des employeurs et tuteurs.

In fine, la transition agro-écologique invite à déplacer le regard des savoirs vers les situations professionnelles qui appellent leur mobilisation. Sa réussite dépend, pour une part, d'une stratégie à négocier par tous les acteurs du processus d'enseignement/apprentissage concernant le choix des situations professionnelles «à apprendre ", et donc à contractualiser avec les tuteurs professionnels. Cette stratégie d'apprentissage suppose de se répartir les situations de formation, les contenus à faire apprendre et aussi une redéfinition des tâches de chacun. A défaut, perdurera le sentiment de certains enseignants de " ramer à contre-courant " d'une vision productiviste, lorsqu'ils enseignent des savoirs relevant, pour eux, de l'agro-écologie. 
Enfin, ce débat sur l'activité implique les normes amont du contrôle de la qualité du travail d'enseignement par les inspecteurs. En effet, la pratique actuelle des enseignants se fonde sur un référentiel qui, s'il peut s'aménager, a vocation à être respecté (en l'occurrence, il cadre et finalise les pratiques en faisant apprendre les contraintes dominantes d'une exploitation). Cette pratique est soutenue par les opportunités offertes par l'établissement (par exemple, l'exploitation agricole attenante) pour faire découvrir, expérimenter, apprendre le " produire autrement ». L'organisation ad hoc de la formation mobilise référentiel, structure organisationnelle de l'établissement et opportunités pédagogiques pour la formation, afin d'accompagner la transition agro-écologique prévue. La tâche attendue des enseignants (Leplat, 1997) est soumise à des normes de contrôle et de renforcement (de la conformité par l'inspection, de la validité par les pairs, de la ratification par les milieux professionnels concernés ; Jarniou, 1981).

Les mises en situations liées à l'enseignement de l'agro-écologie engagent un débat de normes lié autant à la façon d'enseigner cet objet qu'à celle de délimiter la diversité de nature des savoirs, des pratiques agro-écologiques et des représentations associées. Elles questionnent également la pertinence des lieux où apprendre à penser l'agro-écologie, entre les situations d'enseignement organisées en classe, les situations où se former dans l'exploitation du lycée ou dans les exploitations au sein desquelles se déroulent les stages. Enfin, elles cristallisent les débats sur le "savoir à enseigner ", son enseignement et les normes de contrôle de ce dernier. Ce faisant, elles actualisent la norme du " produire autrement " avec les espaces de socialisation professionnelle des élèves (production/famille), par-delà le contexte injonctif de la transition qui se trouve débattu tant dans l'enseignement que dans le milieu professionnel.

\section{Conclusion}

Comment les capacités des élèves de l'enseignement agricole à saisir les changements de pratiques agro-écologiques sont-elles mises en travail à trois niveaux : dans l'enseignement, sur l'exploitation de lycée, lors des périodes chez un exploitant ?

Nous avons tenté ici de montrer que ces différents cadres de formation mobilisent des ajustements conceptuels, socio-épistémiques et pragmatiques réalisés par les acteurs de l'enseignement ; ajustements liés aux représentations et pratiques des professionnels du monde agricole.

Cela nous permet d'identifier des conditions d'un enseignement de pratiques économes en pesticides. Au-delà de la recherche et de la définition de savoirs robustes ${ }^{19}$ (Martinand, 2007) qui soutiendraient les raisonnements à enseigner, des processus sociaux mêlent les relations et les dépendances réciproques. Ils jouent un rôle essentiel entre les acteurs de l'enseignement et les acteurs du monde professionnel. Leur enjeu réside dans la pos-

19. Nous entendons par là des savoirs fiables et efficaces dans leur mobilisation. 
sibilité offerte (ou non) de procéder à des ajustements de pratiques pour atteindre les objectifs de «l'enseigner à produire autrement ".

Les constats de notre étude, relatifs à l'utilisation du système d'enseignement comme levier pour transformer les pratiques professionnelles agricoles (" produire autrement»), se résument en une contradiction : celle de cet objectif avec la volonté de faire avec les acteurs professionnels (les agriculteurs) et d'inscrire les établissements d'enseignement agricole dans leur territoire. En effet, l'analyse que nous avons conduite montre que :

- les enseignants sont conduits à des ajustements conceptuels socio-épistémiques et axiologiques, portant sur les savoirs de la tâche, au regard des représentations qu'ils ont des positions des élèves, parents d'élèves et agriculteurs de leur région concernant la réduction de l'usage des produits phytosanitaires ;

- le rôle assigné aux exploitations agricoles des lycées, et dans le cadre du plan national «Enseigner à produire autrement », se trouve interrogé par les autres acteurs du territoire ; cela invite le directeur de l'exploitation (et les techniciens) à des ajustements conceptuels, socio-épistémiques et pragmatiques, mais aussi technico-gestionnaires, communicationnels, socio-organisationnels, relatifs à l'efficacité des contenus et situations de formation qu'ils proposent ;

- les savoirs agro-écologiques enseignés peuvent être " disqualifiés » dans l'action professionnelle que les élèves rencontrent lors des périodes dans les exploitations agricoles extérieures au lycée, conduisant à les cantonner à la « sphère scolaire ";

- les points précédents invitent les corps d'inspection à réinterroger leurs processus de renforcement et de contrôle.

L'enjeu, pour cet enseignement et ses acteurs, semble alors lié à une capacité à intégrer, dans les situations de formation, ces ruptures conceptuelles (façons de penser), socioépistémiques (façons de comprendre) et pragmatiques (façons de faire) qui traversent le "produire autrement"; et ce, tant dans les contenus que dans les modalités pédagogiques à redéfinir pour aborder, en classe, des débats de normes, la construction historique de controverses, et les débats socio-épistémiques associés.

Au terme de cet article, il convient de souligner la dimension « laboratoire " de l'implantation de cette réforme, entre ruptures et ajustements. Contrairement à ce qui se passe au sein d'autres ministères, le système d'enseignement professionnel agricole bénéficie d'un " circuit court " entre l'injonction politique et sa mise en œuvre dans les classes des lycées. Cette transmission directe est d'un grand avantage si la politique décidée est proche d'un existant connu du politique. Cet avantage est annhilé si ce dernier méconnaît les professionnels, la finalité dominante d'insertion professionnelle poursuivie par les enseignants et par les corps intermédiaires (administration centrale, inspections, directions d'établissements,...). 
Les ruptures et ajustements relevés ici mettent en exergue la résilience du système d'enseignement agricole, le potentiel et l'efficacité de modalités d'apprentissages mobilisables grâce aux exploitations des établissements agricoles et des organismes de développement. Reste à reconnaître et à valoriser cette résilience et son potentiel à absorber l'impact sur les pratiques actuelles, mais aussi à anticiper les actions qui faciliteraient l'implantation (Contandriopoulos, 2008) non d'une politique, mais de pratiques de durabilité.

\section{Bibliographie}

Abric J. (1994), Pratiques sociales et représentations, Paris, PUF.

Aubertot J.-N., Barbier M., Carpentier A., Gril J.-J., Guichard L., Lucas P., Savary S. (2005), Pesticides, agriculture, et environnement. Réduire l'utilisation des pesticides et en limiter les impacts environnementaux, Paris, INRA-CEMAGREF. En ligne sur http:// www.aspro-pnpp.org/wp-content/uploads/2012/01/Rapport-dexpertise-Pesticides.pdf

Bourdieu P. (1980), Le sens pratique, Paris, Les Editions de Minuit.

Callon M. (1986), «Eléments pour une sociologie de la traduction - La domestication des coquilles Saint-Jacques et des marins-pêcheurs dans la baie de Saint-Brieuc ", L'Année Sociologique, $\mathrm{n}^{\circ} 36$, pp. 169-208.

Cancian N., Simonneaux L. (2013), «Construire et analyser des cartographies des controverses sur l'usage des pesticides de synthèse en agriculture en 1983 et en 2007 : enjeux pour l'enquête socio-épistémologique ", Penser l'éducation, Hors série, pp. 503-520.

Contandriopoulos A.-P. (2008), « La gouvernance dans le domaine de la santé : une régulation orientée par la performance ", Santé Publique, vol. 20, nº 2, pp. 191-199 : http:// fulltext.bdsp.ehesp.fr/SantePublique/2008/2/191_200.pdf

Delbos G., Jorion P. (1984), La Transmission des savoirs, Paris, Ed. de la Maison des Sciences de l'Homme.

Direction de l'animation de la recherche, des études et des statistiques (DARES), ministère du Travail, de l'Emploi, de la Formation professionnelle et du Dialogue social (2003), Enquête Sumer 2003.

Direction générale de l'Enseignement et de la Recherche (DGER), ministère de l'Agriculture, de l'Agroalimentaire et de la Forêt (2012). Panorama de l'enseignement agricole 2012 : http://www.chlorofil.fr/fileadmin/user_upload/systeme/organisation/panorama :PANORAMAEA2012.pdf

Douglas M., Wildavsky A. (1983), Risk and Culture. An Essay on the Selection of Technological and Environmental Dangers, University of California Press, Berkeley.

Jarniou P. (1981), L’organisation comme système politique, Paris, PUF. 
Lamine C. (2011), «Anticiper ou temporiser : injonctions environnementales et recompositions des identités professionnelles en céréaliculture ", Sociologie du travail, n 53, pp. 75-92.

Latour B. (1989), La science en action, Paris, La Découverte.

Latour B. (2007), « La cartographie des controverses », Technology Review, n. 0, pp. 82-83.

Legardez A., Simonneaux L. (2006), L'école à l'épreuve de l'actualité : Enseigner les questions vives, Issy-les-Moulineaux, ESF Editeur.

Leplat J. (1997), Regards sur l'activité, Paris, PUF.

Martinand J.-L. (1994), " La didactique des sciences et de la technologie et la formation des enseignants ", Aster, n 19, pp. 61-75.

Martinand J.-L. (2007), "Savoirs robustes et contenus instables en éducation scientifique et technologique ", in Merri M. (coord.), Activité humaine et conceptualisation, Toulouse, Presses Universitaires du Mirail, pp. 203-210.

Mayen P. (2007), «Quelques repères pour analyser les situations dans lesquelles le travail consiste à agir pour et avec un autre ", Recherche en éducation, $\mathrm{n}^{\circ} 4$, pp. 51-64.

Molinié P. (2010), "Souffrance, défenses, reconnaissance. Le point de vue du travail. ", Nouvelle revue de psychosociologie, $\mathrm{n}^{\circ}$ 10, pp. 99-110.

Nallet H. (2013), Concertation sur l'enseignement agricole préparatoire à la loi d'avenir sur l'agriculture, Rapport général, ministère de l'Agriculture, de l'Agroalimentaire et de la Forêt.

Olry P., Astier P. (2001), La mobilisation des savoirs généraux par les apprentis en situation de travail, Rapport au ministère de l'Éducation nationale, DescoA7, Paris, CNAM.

Prévost P. (2013), « Exploitation agricole des établissements d'enseignement, un espace d'expression des capacités d'innovation et d'apprentissage pour la formation des agriculteurs. ", Pour, n 219, pp. 151-159.

Savoyant A. (2008), "Activité et expérience», in Mayen P. et Savoyant A. (eds), Elaboration et réduction de l'expérience dans la validation des acquis de l'expérience, Relief, $\mathrm{n}^{\circ} 28$, Marseille, Céreq, pp. 95-98.

Sebillotte M. (1990), "Système de culture, un concept opératoire pour les agronomes ", in Combe L. et Picard D. (coord.), Les systèmes de culture, Versailles, INRA Edition, pp. 165-196.

Simonneaux J. (2011), Les configurations didactiques des Questions Socialement Vives économiques et sociales, HDR, université de Provence, Aix-Marseille.

Simonneaux L. (2013), "Questions socialement vives and socioscientific issues: New trends of research to meet the training needs of post-modern society ", in Bruguière C., 
Tiberghien A., Clément P. (eds), $9^{t h}$ ESERA Conference Selected Contributions. Topics and trends in current science education, Dordrecht, Springer, pp. 37-54

Teige C. (1993a), "Représentations du travail, travail de la représentation ", in WeillFassina A., Rabardel P., Dubois D. (eds), Représentations pour l'action, Toulouse, Octarès éditions.

Teiger C. et Laville A. (1989), Expression des travailleurs sur leurs conditions de travail (Analyse de sessions de formation de délégués CHS-CT à l'analyse ergonomique du travail), rapport $\mathrm{n}^{\circ} 100$, Collection du Laboratoire d'ergonomie et neurosciences du travail, Paris, Conservatoire national des arts et métiers.

Zask J. (2015), Introduction à John Dewey, Paris, La Découverte. 